\title{
Eficácia da laserterapia de baixa potência em úlceras diabéticas
}

\author{
Efficacy of low power laser therapy in diabetic ulcers \\ Eficacia de la terapia con láser de baja potencia en úlceras diabéticas
}

\section{Resumo}

A fisioterapia, também utiliza - se de recursos tecnológicos para o tratamento de úlceras de pé diabético, tais recursos atuam como modeladores da cicatrização. Como tratamento fisioterapêutico tem se destacado o laser de baixa intensidade com eficácia. Este estudo tem como objetivo buscar evidencias cientificas sobre o uso do laser de baixa intensidade em úlceras em pé diabético. Uma revisão sistemática de literatura, através de buscas em plataformas científicas: Pubmed, SCIELO, LILACS; sendo utilizadas as seguintes palavras chave: fisioterapia; pé diabético, terapia a laser de baixa potência; acatando os seguintes critérios: publicações na língua portuguesa e inglesa, ano de publicação superior a 2010. A análise dos estudos feita através do score de PEDro, demonstrou que os resultados apresentaram evidências de alta qualidade. O laser de baixa intensidade aumenta a expressão de fatores de crescimento que é capaz de induzir a deposição de matriz extracelular, a proliferação celular no local da lesão, favorecendo a angiogênese. A aplicação precoce do laser de baixa potência sobre feridas, se mostrou capaz de acelerar o processo de fechamento das mesmas, com efeitos principalmente nas fases inflamatória e proliferativa. Além disso, o laser atua na estimulação de um processo de cicatrização mais organizado, e tem influência direta na redução do processo antálgico. O presente estudo mostrou que o uso do laser de baixa intensidade usada no tratamento de úlceras diabéticas no processo de aceleração da cicatrização obteve resultados com evidência científica de qualidade elevada, levando em consideração os métodos utilizados e os resultados obtidos, no entanto ainda assim são necessários mais estudos sobre o tema. Palavras-chave: Fisioterapia; Pé diabético; Terapia a Laser de Baixa Potência.

\begin{abstract}
Physiotherapy also uses technological resources for the treatment of diabetic foot ulcers, such resources act as modeling of healing. As a physical therapy treatment, low-intensity laser has been highlighted with effectiveness. This study aims to seek scientific evidence on the use of low-intensity laser in diabetic foot ulcers. A systematic literature review, through searches in scientific platforms: Pubmed, SCIELO, LILACS; the following keywords being used: physical therapy; diabetic foot, low power laser therapy; meeting the following criteria: publications in Portuguese and English, year of publication greater than 2010. The analysis of the studies carried out using the PEDro score showed that the results presented high quality evidence. Low-intensity laser increases the expression of growth factors that are capable of inducing extracellular matrix deposition, cell proliferation at the injury site, favoring angiogenesis. The early application of low-power laser on wounds proved to be able to accelerate the process of wound closure, with effects mainly on the inflammatory and proliferative phases. In addition, the laser acts to stimulate a more organized healing process, and has a direct influence on reducing the antalgic process. The present study showed that the use of low-intensity laser used in the treatment of diabetic ulcers in the healing acceleration process obtained results with high quality scientific evidence, taking into account the methods used and the results obtained, however they are still necessary more studies on the subject.
\end{abstract}

Keywords: Physical therapy specialty; Diabetic foot; Low-Level Light Therapy. 


\begin{abstract}
Resumen
La fisioterapia, sin control de las úlceras del pie diabético, cuenta con recursos físicos que modelan la cicatrización y la La fisioterapia también utiliza recursos tecnológicos para el tratamiento de las úlceras del pie diabético, tales recursos actúan como modelo de curación. Como tratamiento fisioterapéutico, se ha destacado con eficacia el láser de baja intensidad. Este estudio tiene como objetivo buscar evidencia científica sobre el uso de láser de baja intensidad en las úlceras del pie diabético. Una revisión sistemática de la literatura, a través de búsquedas en plataformas científicas: Pubmed, SCIELO, LILACS; se utilizan las siguientes palabras clave: fisioterapia; pie diabético, terapia con láser de baja potencia; cumpliendo los siguientes criterios: publicaciones en portugués e inglés, año de publicación superior a 2010. El análisis de los estudios realizados mediante el puntaje PEDro mostró que los resultados presentaban evidencia de alta calidad. El láser de baja intensidad aumenta la expresión de factores de crecimiento que son capaces de inducir el depósito de la matriz extracelular, la proliferación celular en el sitio de la lesión, favoreciendo la angiogénesis. La aplicación temprana de láser de baja potencia sobre las heridas demostró ser capaz de acelerar el proceso de cierre de la herida, con efectos principalmente en las fases inflamatoria y proliferativa. Además, el láser actúa para estimular un proceso de curación más organizado y tiene una influencia directa en la reducción del proceso antálgico. El presente estudio mostró que el uso de láser de baja intensidad utilizado en el tratamiento de úlceras diabéticas en el proceso de aceleración de la cicatrización obtuvo resultados con evidencia científica de alta calidad, teniendo en cuenta los métodos utilizados y los resultados obtenidos, sin embargo aún son necesarios más estudios sobre el tema.
\end{abstract}

Palabras clave: Especialidad de fisioterapia; Pie diabético; Terapia por Luz de Baja Intensidad.

\title{
1. Introdução
}

Segundo Neto et al. (2017), o diabetes mellitus é considerado um grande desafio de saúde pública, pois sua grande taxa de mortalidade afeta diretamente a qualidade de vida dos indivíduos e gera altos custos financeiros pela longevidade do tratamento.

O diabetes mellitus (DM) se caracteriza como doença crônica dominante a nível global o que gera impactos na vida das pessoas por suas complicações agudas e crônicas e as altas taxas de hospitalizações, repercutindo em um alto porcentual de morbimortalidade, com danos sociais e econômicos (Souza et al., 2017).

Por conseguinte, o DM trata-se de um distúrbio metabólico crônico e degenerativo, manifestado por hiperglicemia crônica, decorrente da destruição das células beta presente no pâncreas, falta de insulina ou incapacidade de exercer adequadamente seus efeitos (Oliveira et al., 2017).

Conforme Macedo et al. (2017), dentro dos agravos crônicos do DM, o pé diabético é a causa mais comum de complicações com altas taxas de amputações e internações prolongadas. O pé diabético é uma complicação do Diabetes mellitus, ocorre quando uma área lesionada ou infeccionada nos pés desenvolve uma úlcera. Seu aparecimento pode ocorrer quando a circulação sanguínea é deficiente e os níveis de glicemia são mal controlados.

De acordo com Brasileiro et al. (2019), a complexidade do DM, os avanços com o laser de baixa intensidade no tratamento de úlceras em pé diabéticos, será abordada nesse estudo, medidas de prevenção são essenciais no controle, orientações como: controle glicêmico, avaliação dos níveis de hemoglobina glicosilada periodicamente, cuidado com os pés, visitas regulares ao médico, assistência da família são importantes para o não agravo da mesma.

O laser de baixa intensidade atua na úlcera do pé diabético aumentado a velocidade do processo de cicatrização e regeneração tecidual, aonde pode ser combinado com outros métodos de tratamentos (Castro et al., 2020).

A palavra laser segundo Salesiano et al. (2020), é oriunda da língua inglesa que significa "light amplification by stimulated emission of radiation" (amplificação de uz por emissão estimulada de radiação). A radiação emitida tem origem eletromagnética não ionizante podendo ou não ser visível. Os comprimentos das ondas utilizadas na laserterapia encontram-se visível no infravermelho, no que emite ondas de $770 \mathrm{~nm}$ até 1550nm e estão compostos por cristais de arseneto de gálio (Ga-As) e pelo arseneto de gálio e alumínio (Ga-Al-As). O arseneto de gálio (Ga-As) emite comprimentos de onda de 904nm com uma potência (de pico) entre 10 e 15 Watts (W) de saída. É invisível, pulsado e possui uma penetração de 30 a $50 \mathrm{~mm}$. O laser de alta potencia ou cirúrgico se beneficia dos efeitos fotoablativo, fotoionizante, fotomecânico-acústico e fototérmico, através de 
vaporização e coagulação local, isso facilita a homeostasia. Em contra partida o laser de baixa potência modula as células por resposta fotobiomolecular por meio de um efeito fotofísico-químico.

O laser de baixa intensidade (LBI) refere-se ao uso de raios vermelhos e infravermelhos com um comprimento de onda entre 600 e $1000 \mathrm{~nm}$ e potência entre 5-500 miliwatts. A luz do laser pode penetrar profundamente nos tecidos, onde ela tem um efeito fotobioestimulante (Souza et al., 2020).

O laser de baixa intensidade (LBI) é um recurso fisioterapêutico explorado há pouco tempo, sendo um método de baixo custo, seguro e não invasivo, evidências cientificas comprovam sua eficácia no processo de cicatrização e regeneração tecidual. Essa terapêutica vem sendo utilizada cada vez mais pelos profissionais de saúde da fisioterapia, se beneficiando dos lasers, os de: HélioNeônio (He-Ne), Arseneto de Gálio (AsGa), Alumínio-Gálio-Indio-Fósforo (AlGaInP) e ArsenetoGálio-Alumínio (AsGaAl), conhecidos como laseres terapêuticos, laseres de baixa intensidade ou de baixa potência. Sua atuação no processo cicatricial se da a partir do aumento do metabolismo, maturação, proliferação celular, maior quantidade de granulação dos tecidos e redução dos mediadores inflamatórios (Nascimento \& Morais, 2020).

O laser de baixa intensidade segundo Jesus et al. (2020), aplicada em ulcerações alavanca os efeitos fisiológicos do organismo como, depósito de colágeno, revascularização local do tecido, proliferação do epitélio, efeitos anti-inflamatórios e analgésicos, ações que combinadas aceleram o efeito de cicatrização das úlceras, o que evita e preveni maiores complicações como, a evolução para uma amputação de membro. A utilização do laser é uma alternativa terapêutica não invasiva, indolor, eficaz e de baixo custo no tratamento, tendo em vista que promoverá uma maior qualidade de vida e bem estar dos pacientes. A presente revisão de cunho sistemático teve como objetivo analisar a eficácia do laser de baixa intensidade no processo de cicatrização de úlceras diabéticas.

\section{Metodologia}

A atual pesquisa classifica-se como revisão sistemática da literatura seguindo as diretrizes e recomendações PRISMA (Moher et el., 2015). De tal maneira, foram incumbidos a averiguações em bases de dados, três pesquisadores independentes entre si. Após a busca as discordâncias entre os elementos foram resolvidas através de discursões em reuniões posteriores.

O estudo em questão buscou responder a situação disposta: a laserterapia de baixa potência apresenta eficácia no tratamento de úlceras diabéticas?

Como modo de formulação da revisão, realizou-se pesquisas nas bases de dados virtuais ligadas a Pubmed. Três pesquisadores individuais efetuaram o atual estágio do estudo. Os descritores utilizados foram: "low power laser" e "therapy diabetic ulcers", além da combinação dos termos entre si. O tempo de arrecadação de dados persistiu de janeiro até setembro de 2021.

Como modo de constituição do presente estudo os artigos necessitariam respeitar tais critérios de elegibilidade: artigos publicados na língua inglesa, com período de tempo entre 2011 a 2021, ensaios clínicos randomizados, com escore maior que 6 na escala de qualidade PEDro, e que apresentassem a eficácia da laserterapia de baixa potência no tratamento de úlceras diabéticas. Foram exclusos estudo de casos, revisão bibliográficas, comentários clínicos, além de artigos que não retratassem o uso do laser de baixa intensidade em úlceras diabéticas.

As variáveis consideradas nos artigos foram: título do artigo, data de publicação, amostra, descrição da amostra, grupos de pesquisas, características do grupo, caracterização do laser, metodologia de avaliação, período de tratamento, resultados, pontuação na escala de qualidade PEDro. 


\section{Resultados e Discussão}

Os estudos escolhidos foram analisados de acordo com a escala de qualidade PEDro. A mesma qualifica as características dos artigos exteriorizados conforme 11 itens. Item 1 equivale a sua legitimidade externa do estudo, Itens 2 a 9 equivalem a sua legitimidade interna, em contrapartida os itens de 10 a 11 equivalem a peculiaridade metodológica estatística do estudo. É conferido 1 ponto para cada fundamento completo, sem pontuar o item número 1, o que totalizará 10 pontos. Julgase importante para a análise dos critérios da escala PEDro determinada pontuação: 0-3: estudos de baixa qualidade; 4-5: média qualidade; 6-10 alta qualidade (Shiwa et al., 2011). O compilado dos resultados obtidos através da análise de qualidade dos estudos está disposta na Tabela 1 .

Tabela 1. Caracterização dos artigos de acordo com a evidência científica pelo SCORE de PEDro.

\begin{tabular}{|c|c|c|c|c|c|c|}
\hline AUTOR & AMOSTRA & $\begin{array}{l}\text { GRUPOS } \\
\text { DE } \\
\text { ESTUDO }\end{array}$ & $\begin{array}{c}\text { CARACTERÍSTICAS } \\
\text { DO LASER }\end{array}$ & $\begin{array}{l}\text { MÉTODO DE } \\
\text { AVALIAÇÃO }\end{array}$ & $\begin{array}{l}\text { PERÍODO DE } \\
\text { TRATAMENT } \\
\text { O }\end{array}$ & $\begin{array}{l}\text { PONTUAÇÃO } \\
\text { PEDro }\end{array}$ \\
\hline $\begin{array}{l}\text { Carvalho } \\
\text { et al } 2016\end{array}$ & 32 & $\begin{array}{c}\mathrm{GC} / \mathrm{GL} / \mathrm{GC} \\
\mathrm{O} / \mathrm{GC}\end{array}$ & $\begin{array}{c}658 \mathrm{~nm} / 80 \text { segundos} / \\
4 \mathrm{j} / \mathrm{cm}^{2}\end{array}$ & $\begin{array}{l}\text { Ultrassom doppler/ } \\
\text { EVA/ Área da ferida }\end{array}$ & $\begin{array}{c}30 \text { dias/ } 3 \\
\text { encontros } \\
\text { semanais } 12 \\
\text { sessões } \\
\end{array}$ & 7 \\
\hline $\begin{array}{l}\text { Feitosa et } \\
\text { al } 2015\end{array}$ & 16 & GC/GLLLT & $\begin{array}{c}632,8 \mathrm{~nm} / 80 \\
\text { segundos } / 4 \mathrm{j} / \mathrm{cm}^{2}\end{array}$ & Área da ferida/EVA & $\begin{array}{c}30 \text { dias/12 } \\
\text { encontros/ } 3 \\
\text { sessões semanais }\end{array}$ & 6 \\
\hline $\begin{array}{c}\text { De } \\
\text { Alencar } \\
\text { et al } 2018\end{array}$ & 18 & GC/GL & $\begin{array}{c}\text { 660nm/potência } 30 \\
\mathrm{mw} / 6 \mathrm{j} / \mathrm{cm}^{2}\end{array}$ & $\begin{array}{c}\mathrm{PUSH} / \mathrm{EVA} / \text { escala da } \\
\text { ferida }\end{array}$ & 4 semanas $/ 48 / 48 \mathrm{~h}$ & 6 \\
\hline $\begin{array}{l}\text { Mathur et } \\
\text { al } 2016\end{array}$ & 30 & GC/GLLLT & $\begin{array}{c}660+20 \mathrm{~nm} / 60 \text { segundos} / \\
3 \mathrm{j} / \mathrm{cm}^{2}\end{array}$ & $\begin{array}{c}\text { Área da } \\
\text { ferida/vascularização/do } \\
\text { ppler colorida/ }\end{array}$ & 15 dias & 6 \\
\hline $\begin{array}{l}\text { Johnson } \\
\text { et al } 2019\end{array}$ & 34 & GC/GP/GL & $\begin{array}{l}2940 \mathrm{~nm} / 30 \text { segundos} / 25 \mathrm{j} \\
/ \mathrm{cm}^{2}\end{array}$ & $\begin{array}{c}\text { Área da } \\
\text { ferida/desbridação/cicat } \\
\text { rização }\end{array}$ & 60 dias & 7 \\
\hline $\begin{array}{l}\text { Vitoriano } \\
2019\end{array}$ & 12 & $\begin{array}{l}\text { Gblaser/Gbl } \\
\text { ed }\end{array}$ & $\begin{array}{c}830 \mathrm{~nm} / 30 \mathrm{~mW} / 8,4 \mathrm{w} \\
\text { potência } / \mathrm{cm}^{2}\end{array}$ & $\begin{array}{c}\text { Área da ferida/avaliação } \\
\text { da neuropatia }\end{array}$ & $\begin{array}{c}10 \text { sessões/e } \\
\text { vezes por semana }\end{array}$ & 6 \\
\hline $\begin{array}{l}\text { Ruh et al } \\
2018\end{array}$ & 8 & GD/GUP & $660 \mathrm{~nm} / 100 \mathrm{mw} / 2 \mathrm{j} / \mathrm{cm}^{2}$ & Área da ferida & 12 dias & 7 \\
\hline
\end{tabular}

Fonte: Oliveira, Abreu, Cavalcante e Silva (2021).

A análise dos estudos feita através do score de PEDro, demonstrou que os 7 estudos apresentaram evidências de alta qualidade. De acordo com Shiwa et al. (2011), a aplicação de intervenções que são baseadas em evidências científicas é necessária para que os pacientes que são alvo de tais intervenções, recebam tratamentos que sejam de fato eficazes, o que impacta diretamente na redução de custos de saúde em geral e qualidade de vida.

Para a comunidade acadêmica, serve de base para a implementação e adaptação de protocolos nas diferentes realidades, em relação estudo em questão, para o uso do laser de baixa potência em lesões diabéticas. Diante disso se pode identificar a importância de estudos que apresentem resultados objetivos bem definidos, além de resultados descritos de forma correta e coerente, baseados em uma metodologia padronizada e sistemática. A Tabela 2 apresenta um compilado dos resultados e conclusões a respeito da eficácia do laser de baixa potência em úlceras diabéticas, dos estudos analisados nesta obra. 
Tabela 2. Eficácia do uso do Laser de Baixa Potência em úlceras diabéticas.

\begin{tabular}{|c|c|c|}
\hline AUTOR & RESULTADOS & CONCLUSÕES \\
\hline Carvalho et al 2016 & $\begin{array}{l}\text { Foi observado a redução significativa na dor nos grupos laser e grupo } \\
\text { combinado (laser + calêndula) } \mathrm{p}<0,01 \text {, na avaliação da estrutura do } \\
\text { tendão por meio de ultrassom doppler não foi observador diferenças } \\
\text { entre os grupos. Foi observada a redução da área da ferida nos grupos } \\
\text { laser e combinados (laser + calêndula) } \mathrm{p}<0,01\end{array}$ & $\begin{array}{l}\text { O laser é eficaz na redução da dor e } \\
\text { na área da ferida }\end{array}$ \\
\hline Feitosa et al 2015 & $\begin{array}{l}\text { Percebeu-se a redução do tamanho da ferida e da dor, teve o aumento } \\
\text { significativo de reparação tecidual no grupo LLLT }\end{array}$ & $\begin{array}{l}\text { O laser de baixa potência apresenta } \\
\text { aplicabilidade na redução da área da } \\
\text { ferida e da dor, como também na } \\
\text { melhora do índice de reparação } \\
\text { tecidual }\end{array}$ \\
\hline De Alencar et al 2018 & $\begin{array}{l}\text { Foi observado o aumento significativo no índice de reparo tecidual } \\
\text { no grupo laser, significativa diferença entre os grupos por meio da } \\
\text { escala PUSH,em ambos os grupos não foi observado redução da dor }\end{array}$ & $\begin{array}{l}\text { O laser é eficaz no aumento do } \\
\text { reparo tecidual, na redução da área } \\
\text { da ferida }\end{array}$ \\
\hline Mathur et al 2016 & $\begin{array}{l}\text { O desfecho primário foi a redução absoluta e relativa do tamanho da } \\
\text { ferida em } 2 \text { semanas em comparação com o parâmetro da linha de } \\
\text { base. A redução percentual da área da úlcera foi } 37 \pm 9 \% \text { no grupo } \\
\text { LLLT e } 15 \pm 5,4 \% \text { no grupo controle }(p<0,001) \text {. Para } \sim 75 \% \text { das } \\
\text { feridas do grupo de tratamento, foi observada redução da área da } \\
\text { ferida de } 30-50 \% \text {. Em contraste, para o grupo de controle, } \sim 80 \% \\
\text { das feridas mostraram uma redução da área da ferida de }<20 \% \text { no dia } \\
15 \text {. }\end{array}$ & $\begin{array}{l}\text { Os resultados sugerem que a LLLT } \\
\text { é benéfica como um complemento à } \\
\text { terapia convencional no tratamento } \\
\text { de úlceras do pé diabético. }\end{array}$ \\
\hline Johnson et al 2019 & $\begin{array}{l}\text { O tratamento pós-laser de } 4 \text { semanas PWAR foi significativamente } \\
\text { maior do que antes do tratamento com laser (intervalo de confiança } \\
\text { de } 95 \%-152,7 \mathrm{a}-41,2 ; \mathrm{p}=0,002) \text {. Dezesseis }(72,7 \%) \text { de } 22 \text { feridas } \\
\text { alcançaram> } 50 \% \text { de WAR em } 4 \text { semanas. Onze }(50 \%) \text { das } 22 \\
\text { feridas foram completamente curadas em ou antes } 12 \text { semanas. } \\
\text { Nenhuma das } 6 \text { feridas com }<50 \% \text { WAR em } 4 \text { semanas foi curado } \\
\text { em } 12 \text { semanas. }\end{array}$ & $\begin{array}{l}\text { A terapia a laser Erbium: YAG } \\
\text { acelerou a cura do DFU em uma } \\
\text { coorte de pacientes com úlceras que } \\
\text { não responderam à terapia padrão. }\end{array}$ \\
\hline Vitoriano et al. 2019 & $\begin{array}{l}\text { Em relação ao grupo laser, foi observada redução da extensão da } \\
\text { ferida em } 79,43 \% \text { ao final da } 10^{a} \text { sessão; os pacientes do grupo LED } \\
\text { tiveram redução de } 55,84 \% \text { no processo de } \\
\text { cicatrização; comparando as duas terapias observou-se uma melhor } \\
\text { cicatrização nos participantes do grupo laser, com } 81,17 \% \text {, em } \\
\text { relação ao LED após o término das sessões; em relação à avaliação } \\
\text { do quadro neuropático, houve melhora significativa em ambas as } \\
\text { terapias. }\end{array}$ & $\begin{array}{l}\text { Houve melhora dos sinais e } \\
\text { sintomas neuropáticos, também } \\
\text { melhora da reparação tecidual nas } \\
\text { duas modalidades terapêuticas; } \\
\text { entretanto, o laser apresentou um } \\
\text { índice de velocidade maior em } \\
\text { relação ao LED. }\end{array}$ \\
\hline Hur et al. 2018 & $\begin{array}{l}\text { LLLT promoveu uma melhora da aparência macroscópica dos graus } \\
\text { III e IV de PS. } \\
\text { - VEGF e TGF- } \beta \text {, fatores de crescimento relacionados à angiogênese } \\
\text { tecidual, aumentaram após o tratamento. } \\
\text { - IL-6 não mudou após o tratamento. } \\
\text { - TNF reduzido após o tratamento. }\end{array}$ & $\begin{array}{l}\text { Após a LLLT, as feridas } \\
\text { apresentaram melhora do aspecto } \\
\text { macroscópico, com aumento dos } \\
\text { fatores VEFG e TGF- } \beta \text {, e redução } \\
\text { do TNF; apesar de nossos } \\
\text { resultados promissores, eles devem } \\
\text { ser analisados com cuidado, pois } \\
\text { este estudo não teve um grupo de } \\
\text { controle. }\end{array}$ \\
\hline
\end{tabular}

Fonte: Oliveira, Abreu, Cavalcante e Silva (2021).

Santos et al. (2011), apresenta os benefícios da aplicação do laser de baixa potência, dentre os quais se destacam os seguintes: aceleração do processo de reparo tecidual, efeitos analgésicos, anti-inflamatórios e de regeneração tecidual. Dos estudos analisados $100 \%$ apresentaram como resultado a reparação tecidual.

O laser de baixa intensidade aumenta a expressão de fatores de crescimento, como TGF- $\beta$, que, por sua vez, é capaz de induz deposição de matriz extracelular, incrementando a proliferação celular no local da lesão, aumentando a quantidade de vasos sanguíneos (processo chamado de neogênese) promovendo assim a reparação tecidual (Rocha et al., 2012).

Em relação a cicatrização ou diminuição das áreas das lesões diabéticas, as literaturas destacaram a acentuada redução da área da lesão ao longo das sessões com o laser (Alencar et al., 2018; Carvalho et al., 2016; Feitosa et al., 2015). A aplicação 
precoce do laser de baixa potência sobre feridas, se mostrou capaz de acelerar o processo de fechamento das mesmas, com efeitos principalmente nas fases inflamatória e proliferativa. Além disso, o laser atua na estimulação de um processo de cicatrização mais organizado, que influencia diretamente no aspecto estético da cicatriz, impedindo a formação de cicatrizes grosseiras (Ferreira, 2016).

Outro fator evidenciado foi redução do processo antálgico (Carvalho et al., 2016; Feitosa et al., 2015). Uma das principais características da dor patológica é a hipersensibilização, que são decorrentes das alterações na plasticidade do sistema nervoso, que ocorre perifericamente por redução do limiar de ativação dos nociceptores ou de forma central, pela responsividade aumentada da medula espinhal aos estímulos sensoriais (Janeiro, 2017).

A respeito deste achado, Sanchez, Andrade e Parizotto (2018), corroboram que os efeitos analgésicos induzidos pela LLLT podem ser justificados através da modulação dos mediadores químicos da inflamação, juntamente com o estímulo à síntese de beta endorfina. Essa associação de fatores são responsáveis por limitar a redução do limiar de excitabilidade dos receptores dolorosos e eliminar substâncias alogênicas.

Em contraponto, Janeiro (2017) destacou que alguns pesquisadores e terapeutas vem questionado os benefícios clínicos do laser de baixa potência devido aos resultados divergentes encontrados. Porém, isto pode se justificar pelo que de haver carência na padronização metodológica nos estudos, como se pôde observar nas metodologias expostas no presente estudo.

\section{Considerações Finais}

De um modo geral, o paciente diabético requer condutas multiprofissionais para cura ou cicatrização da úlcera, melhora nas condições clínica e psicossocial. Uma técnica usada isoladamente, a custo, com dificuldade terá aptidão de estimular a cura por completa. Para maiores eficácias, cabe à equipe multiprofissional buscar melhores evidencias na definição de seu tratamento. O presente estudo mostrou que o uso do laser de baixa intensidade usada no tratamento de úlceras diabéticas no processo de aceleração da cicatrização obteve resultados com evidência científica de qualidade elevada, levando em consideração os métodos utilizados e os resultados obtidos, no entanto ainda assim são necessários mais estudos sobre o tema. Não se teve a pretensão de esgotar os conhecimentos a respeito do tema proposto, mas sim para que esta obra sirva de fomento para estudos de campo, revisões de literaturas e base para construção de manuais e protocolos relacionados ao uso do laser de baixa potência em lesões de difícil cicatrização.

\section{Agradecimentos}

Agradecemos primeiramente a Deus pela saúde e coragem que nos tem dado durante esses cincos anos de graduação, agradecemos também ao nosso orientador Wanderson, pois sem ele nada disso seria possível, nos e orientou tão bem durante esses dois últimos semestres da graduação, não só com conhecimento, mas também pelo incentivo, nos encorajou como ninguém. Somos gratos a tamanha dedicação.

\section{Referências}

Andrade, S. M., Stefano, S. R., \& Zampier, M. (2017). Metodologia de pesquisa. UNICENTRO. 7-8.

Brasileiro, J. L., Oliveira, W. T. P., Monteiro, L. B., Chen, J., Pinho Jr, E. L., Molkenthin, S., \& Santos, M. A. (2019) Pé diabético: aspectos clínicos. Sociedade Brasileira de Angiologia e Cirurgia Vascular. Jornal vascular brasileiro, 4 (1), 11-21.

Carvalho, A. F. M., Feitosa, M. C. P., Coelho, N. P. M. F., Rebêlo, V. C. N., Castro, J. G., Sousa, P. R. G., Feitosa, V. C., \& Arisawa, E. A. S. (2016) Low-level laser therapy and Calendula officinalis in repairing diabetic foot ulcers. Revista Escola Enfermagem USP. 50(4):628-634.

De Castro, M. F., Barbosa, L. R. P., \& Da Silva, L. L. (2020). Ação da terapia a laser de baixa intensidade na cicatrização de ulcerações diabéticas. Research, Society and Development, 9(10), e6239109109-e6239109109. 
De Jesus, P. A. P. Nascimento, K. F., Martins, A. C. A., Silva, N. M. P., \& Azevedo, M. V. G. T. (2020). Utilização Do Laser Na Cicatrização Do Pé Diabético. Reveista Unilus Ensino E Pesquisa, 16(45), 311-318.

De Sousa, L. S. N., Rodrigues, M. T. P., Mascarenhas, M. D. M., \& Silva, A. R. V. (2017) Conhecimento do enfermeiro sobre a prevenção do pé diabético: revisão integrativa da literatura. Revista Brasileira em Promoção da Saúde, 30(3). 10.5020/18061230.2017.6602

Feitosa, M. C. P., Carvalho, A. F. M., Feitosa, V. C., Coelho, I. M., Oliveira, R. A., \& Arisawa, E. Â. L. (2015). Effects of the Low-Level Laser Therapy (LLLT) in the process of healing diabetic foot ulcers. Acta Cirurgia Brasileira. 30(12):852-7.

Ferreira, A. G. A. (2016). Aplicação Do Laser De Baixa Intensidade No Processo De Cicatrização De Ferida Cirúrgica: Padronização Dos Parâmetros Dosimétricos. Universidade Federal De Minas Gerais Programa De Pós-Graduação Em Engenharia Mecânica. https://repositorio.ufmg.br/bitstream/1843/BUBD-AC3LL8/1/vers_o_final_disserta_o_aline_gomes_afonso_ferreira_1_.pdf.

Janeiro, Inês M. I. (2017). Fisiologia Da Dor. Universidade Lusófona de Humanidades e Tecnologias Escola de Ciências e Tecnologias da Saúde. https://recil.grupolusofona.pt/bitstream/10437/890 4/1/Fisiologia\%20da\%20dor\%20-\%20Vers\%C3\%A3o\%20Final\%20-\%202017.pdf.

Johnson, M. J., Crisologo, P. A., Truong, D. H., Wukich, D. K., Oz, O. K., Fontaine, J., \& Lavery, L. A. (2019). Erbium: Yttrium Aluminum Garnet Laser Accelerates Healing in Indolent Diabetic Foot Ulcers J Foot Ankle Surg. 58(6):1077-1080. 10.1053/j.jfas.2019.07.023.

Macedo, J. L. Pereira, I. C., Oliveira, Amanda, S. S. S., \& Magalhães, M., J. S. (2017). Eficácia da fitoterapia no processo de cicatrização tecidual de pacientes com diagnóstico de diabetes mellitus. Revista Ciência \& Saberes-UniFacema, 3(1), 396-400.

Mathur, R. K., Sahu, K., Saraf, S., Patheja, P., Khan, Fareed., \& Gupta, P. K. (2016). Low-level laser therapy as an adjunct to conventional therapy in the treatment of diabetic foot ulcers Lasers. Medicine Science. 32(2):275-282. 10.1007/s10103-016-2109-2.

Moher, D., Liberati, A., Tetzlaff, J., \& Altman, D. G. The PRISMA Group. (2015). Principais itens para relatar Revisões sistemáticas e Meta-análises: A recomendação PRISMA*. Epidemiologia. Serviço de Saúde, 335. 24(2): 10.5123/S1679-49742015000200017.

Nascimento, I. A. C., \& Morais, R. R. F. (2020). A utilização do laser na cicatrização de úlceras venosas: revisão sistemática.

Neto, M. O., Pereira, M. S.; Pinto, M. A. H.; Agostinho, L. M.; Reinaldo Júnior, F. E.; \& Hissa, M. N. (2017). Avaliação do autocuidado para a prevenção do pé diabético e exame clínico dos pés em um centro de referência em diabetes mellitus. Journal of Health \& Biological Sciences, 5(3), $265-271$.

Oliveira, A. F., Marchi, A. C. B., Leguisamo, C. P., Baldo, G. V. \&, Wawginiak, Thiago, A. (2017). Estimativa do custo de tratar o pé diabético, como prevenir e economizar recursos. Ciência \& Saúde Coletiva, 19(6), 1663-1671. 2014. < http://www.scielo.br/pdf/csc/v19n6/1413-8123-csc-19-06-01663.pdf>.

Rocha, C. L. J. V., Rocha Júnior, A. M., Aarestrup, B. J., \& Aarestrup, F. M. (2012). Inibição da expressão de ciclooxigenase 2 em feridas cutâneas de camundongos submetidos à terapia a laser de baixa intensidade. Jornal Vascular Brasileiro, 11(3), 175-181.

Ruh, A. C., Frigo, L., Cavalcanti, M. F. X. B., Svidnicki, P., Vicari, V. N., Martins, Rodrigo, A. B. L., Junior, E. C. P. L., Isla, N., Diomede, F., Trubiani, O., \& Favero, G. M. (2017). Laser photobiomodulation in pressure ulcer healing of human diabetic patients: gene expression analysis of inflammatory biochemical markers Lasers Medicine Science. 2018 Jan;33(1):165-171. 10.1007/s10103-017-2384-6.

Salesiano, Centro Universitário C. et al. Análise Dos Efeitos Analgésicos Da Fotobiomodulação A Laser Infravermelho Em Tratamento Fisioterapêutico: Uma Revisão Sistemática. LINS - SP 2020.

Sanchez, A. D., Andrade, A. L. M., \& Parizotto, N. A. (2018). Eficácia da terapia a laser de baixa intensidade no controle da dor neuropática em camundongos. Fisioterapia Pesquisa. ;25(1):20-27. 10.1590/1809-2950/16557525012018.

Santos, J. A. F., Campelo, M. B. D., Oliveira, R. A., Nicolau, R. A., Rezende, V. E. A., \& Arisawa, E. Â. L. (2018). Effects of Low-Power Light Therapy on the Tissue Repair Process of Chronic Wounds in Diabetic Feet. 10.1089/pho.2018.4455.

Santos, N. R. S., Sobrinho, J. B. M., Almeida, P. F., Ribeiro, A. A., Cangussú, M. C. T., Santos, J. N., \& Pinheiro, A. L. B. (2011). Influence of the combination of infrared and red laser light on the healing of cutaneous wounds infected by staphylococcus aureus. Photomedicine and Laser Surgery, 29(3),.177-182.

Sassim, P. V. S., Ferreira, T. C. R., Pena, J. C. V., Lima, P. T. S., \& Lima, N. T. O. (2020). Efeitos Do Laser De Baixa Intensidade No Tecido Muscular. Revisão Sistemática. Revista CPAQV-Centro de Pesquisas Avançadas em Qualidade de Vida-CPAQV Journal, 12 (2).

Shiwa, S. R., Costa, L. O. P., Moser, A. D. L., Aguiar, I. C., \& Oliveira, L. V. F. (2011). PEDro: a base de dados de evidências em fisioterapia. Fisioterapia. Movimento., 24(3), 523-533.

Vitoriano, N. A. M., Mont'Alverne, D. G. B., Martins, M. I. S., Silva, P. S., Martins, C. A., Teixeira, H. D., Miranda, C. B., Bezerra, L. M. M., Montenegro Jr, R. M., \& Rocha, J. C. T. (2019). Comparative study on laser and LED influence on tissue repair and improvement of neuropathic symptoms during the treatment of diabetic ulcers Lasers. Medine Scienc. 34(7):1365-1371. 10.1007/s10103-019-02724-5. 